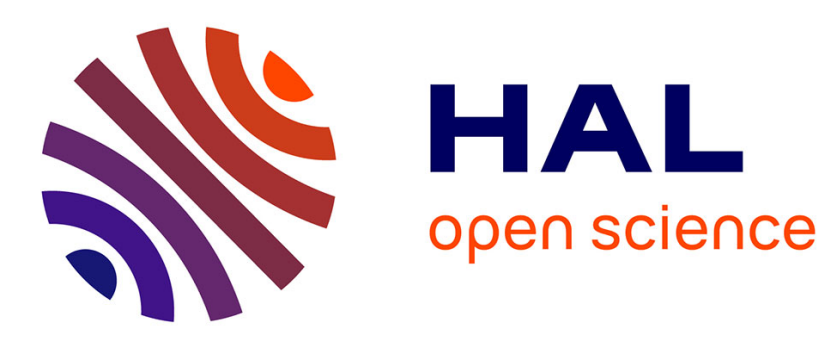

\title{
Chambre à étincelles cylindrique à fils avec enregistrement par ferrites
}

A.E. Taylor, I. Blair, A. Astbury, D. Axen, P. Kalmus, M. Gouanère

\section{To cite this version:}

A.E. Taylor, I. Blair, A. Astbury, D. Axen, P. Kalmus, et al.. Chambre à étincelles cylindrique à fils avec enregistrement par ferrites. Revue de Physique Appliquée, 1969, 4 (2), pp.304-305. 10.1051/rphysap:0196900402030400 . jpa-00243273

\section{HAL Id: jpa-00243273 https://hal.science/jpa-00243273}

Submitted on 1 Jan 1969

HAL is a multi-disciplinary open access archive for the deposit and dissemination of scientific research documents, whether they are published or not. The documents may come from teaching and research institutions in France or abroad, or from public or private research centers.
L'archive ouverte pluridisciplinaire HAL, est destinée au dépôt et à la diffusion de documents scientifiques de niveau recherche, publiés ou non, émanant des établissements d'enseignement et de recherche français ou étrangers, des laboratoires publics ou privés. 


\title{
GHAMBRE A ÉTINCELLES GYLINDRIQUE A FILS AVEG ENREGISTREMENT PAR FERRITES
}

\author{
A. E. TAYLOR, I. BLAIR, \\ Atomic Energy Research Establishment, \\ A. ASTBURY, D. AXEN, P. KALMUS, \\ Rutherford High Energy Laboratory, Chilton, \\ et M. GOUANÈRE, \\ Institut de Physique Nucléaire, Université de Lyon (France).
}

\begin{abstract}
Résumé. - Dans une expérience à taux de comptage faible, on a utilisé des chambres à étincelles cylindriques à fils, avec enregistrement par ferrites, présentant l'avantage d'une quantité de matériau réduite pour diminuer le bruit de fond dû à des réactions parasites.

Abstract. - We have used cylindrical wire spark chambers with a core reading system in a low counting experiment. These chambers leave a small amount of material in the beam, to reduce the background due to other reactions.
\end{abstract}

L'expérience dans laquelle nous avons utilisé ces chambres a pour but la mesure du paramètre d'asymétrie, dans la désintégration leptonique :

$$
\Sigma^{-} \rightarrow \mathrm{n}+\mathrm{e}^{-}+\nu
$$

conduisant à une valeur du rapport $g \mathrm{~A} / g \mathrm{~V}$ de l'interaction faible (fig. 1). La réaction de production est

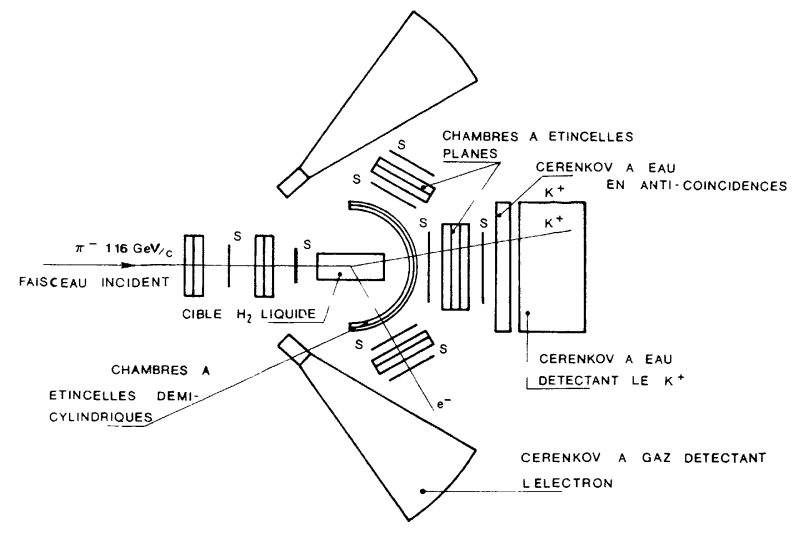

FIG. 1. - Schéma de l'expérience : $\mathrm{S}=$ Détecteur à scintillations.

$\pi^{-}+\mathrm{p} \rightarrow \Sigma^{-}+\mathrm{K}^{+}$. Le schéma de l'expérience est donné par la figure 1 .

La désintégration conduit à un nombre d'événements d'environ 10 par jour, pour une intensité moyenne de $\pi^{-}$de $3 \times 10^{5} / \mathrm{s}$ (soit une intensité instantanée de $\left.3 \times 10^{6} / \mathrm{s}\right)$. Les problèmes de bruit de fond deviennent alors critiques; en particulier, la présence de matériau autour de la cible contribue au déclenchement sur des événements parasites tels que :

$$
\pi^{-}+\mathrm{p} \rightarrow \pi^{-}+\pi^{0}+\mathrm{p}
$$

I. Détection. - Le système détecteur est composé de compteurs à scintillation et de compteurs Čerenkov pour la discrimination des particules. Le $\mathrm{K}^{+}$est détecté dans un tank à eau de 2001 et le $\mathrm{e}^{-}$par un Čerenkov à fréon avec un seuil de $12 \mathrm{MeV}$. La localisation des particules est assurée par des chambres à étincelles à ferrites. Les électrodes sont constituées par des feuilles d'aluminium de $25 \mu \mathrm{m}$ (H.T.) et des grilles de fibre de verre tressées de fils de bronze au phosphore pour l'électrode de masse. Les fils sont distants de 1,2 $\mathrm{mm}$ et ont un diamètre de $0,1 \mathrm{~mm}$.

II. Chambres à étincelles cylindriques ( $f g .2$ ). Les chambres à étincelles disposées après la cible d'hydrogène liquide sont demi-cylindriques, afin de diminuer la présence de matériaux contribuant aux réactions parasites. Un demi-cylindre d'acier de un centimètre d'épaisseur sert de matrice, sur laquelle sont collés des éléments de plexiglas servant de cadres aux électrodes. Des pièces latérales demi-cylindriques servent à tendre les électrodes. Les fils de bronze peuvent être alignés suivant l'axe du cylindre, ou dans la direction perpendiculaire; ce sont alors les fibres de verre qui sont tendues. On a utilisé quatre chambres sur un premier support ( $27 \mathrm{~cm}$ de diamètre) et deux autres chambres sur un support concentrique $(40 \mathrm{~cm}$ de diamètre). Les fils sont reliés aux matrices de ferrites par 
des rallonges de $2 \mathrm{~m}$ de long. Les chambres cylindriqucs ont fonctionné avec les mêmes caractéristiques que lcs chambres planes, c'est-à-dire un intervalle de $6 \mathrm{~mm}$ et une tension de $4,5 \mathrm{kV}$. Il y a en moyenne 4 à 6 traces par événement et l'efficacité dans ces conditions était de $70 \%$ après la reconstruction cinématique et les critères de sélection dans le programme de dépouillement sur IBM 360/75, contre $90 \%$ pour les chambres planes ayant 2 à 3 traces par événement.

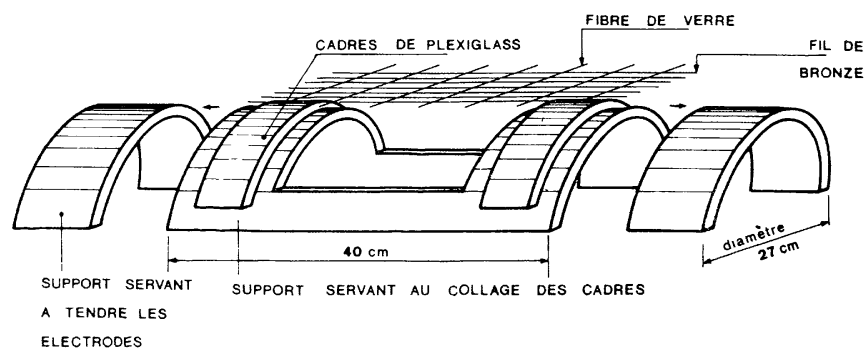

FIG. 2. - Chambres cylindriques.

III. Acquisition des données ( $f i g .3)$. - L'ensemble des chambres à étincelles planes et cylindriques totalise environ 30000 ferrites. Les ferrites sont regroupées sur des matrices de 256 ferrites. Il y a 120 matrices; $100 \mu$ s après l'événement, les ferrites sont lues sur chaque matrice, par groupe de 32 en 8 fois chacune. L'enre-

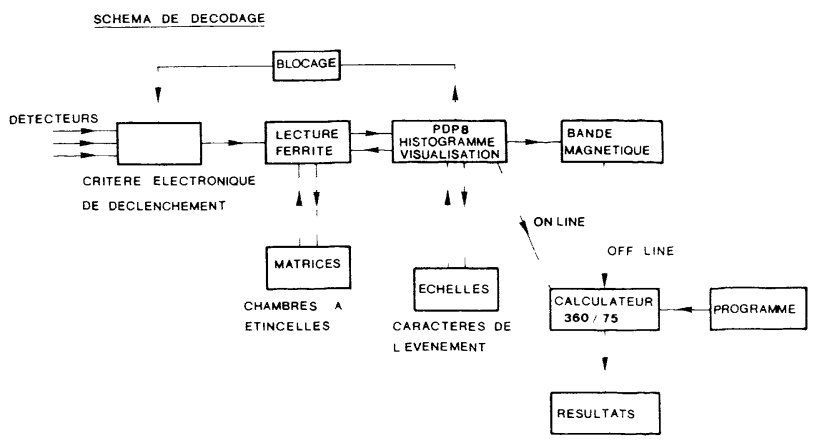

FIG. 3.

Acquisition des données. Schéma de décodage.

gistrement d'un événement est effectué sur bande magnétique par l'intermédiaire d'un PDP 8 . Il comporte les coordonnées des ferrites qui ont été sensibilisées, et les informations provenant d'échelles de comptage au maximum de 14 . A chaque événement, environ 300 ferrites basculent, ce qui conduit à un temps d'enregistrement de $3 \mathrm{~ms}$ (10 $\mu$ s par mot).

L'ensemble réalisé, chambre à étincelles, matrices à ferrites, enregistrement, programme de dépouillement, a été désigné dans l'optique d'une standardisation adaptable à diverses expériences avec seulement des modifications dans les dimensions des chambres. 